\title{
Information and Communication Flows through Community Multimedia Centres: Perspectives from Mozambican Communities.
}

\author{
Community Multimedia Centres (CMCs) are considered by initiating agencies as \\ instruments able to inform, entertain and educate the population, as well as to \\ offering them a voice into the knowledge society and to public initiatives.
}

This article presents a quali-quantitative content analysis of 230 interviews held with staff members, users of the venues, people of the community who listen to their radio component but do not use their telecentres, and community members not using CMCs. The sample includes 10 CMCs around Mozambique. The purpose of the study is to investigate the perception of local communities of inbound, outbound, and shared information and communication flows connected to CMCs.

Results highlight how CMCs are perceived as inbound information enablers, mostly by means of their community radio component, and as means to share information and communication within the communities' boundaries. Yet, CMCs still do not appear to be widely recognized as participation means to a reality that transcend the communities’ physical borders.

Keywords: telecentres, community radios, community multimedia centres, Mozambique, qualitative research, social representations.

\section{Introduction}

Community Multimedia Centres (CMCs) are community-based public access venues (PAV) that combine a community radio with a telecentre. Their primary aim is to foster equitable access to information and knowledge for development, reduce the digital divide, promote social inclusion and civil participation, and promote the circulation of content that supports communities in improving their daily life conditions (Creech, 2006). The official documents of the model of CMCs emphasize the dimensions of information and communication among its grounding aspects, and underline how information and communication should be catalysed and reinforced by the synergy 
among the two components of the radio and the telecentre (UNESCO, 2004). Through CMCs, local community members can access and use a number of different Information and Communication Technologies (ICTs), such as computers, the Internet, digital libraries, fax, photocopy machines, etc., and can listen and participate in a local radio station that is managed by local people and broadcasts community relevant information in native and national languages.

The CMC programme was established by UNESCO in 2001 in Africa, Asia and the Caribbean, and its scale-up phase was launched at the World Summit of the Information Society of Geneva in 2003 (WSIS, 2003). Three African countries, including Mozambique, were selected for this initiative due to the success of a previous pilot phase. Supported, among others, by the Swiss Agency for Development and Cooperation (SDC), the scale-up phase aimed to build 50 CMCs in the following years (UNESCO, 2004). In 2010, the Mozambican Ministry of Science and Technology (MCT) took charge of the programme, with the goal of creating one CMC in each of the 128 districts of the country in the following five years (MCT, 2008). This programme made of CMCs a strategic contribution to achieving the national development and ICT goals of the country. In 2011, 34 CMCs were in place all over the country. Despite inconsistencies in resources and services offered, CMCs remain the prevailing typology of PAV in Mozambique (Rega et al., 2011).

Based on the theoretical framework of Social Representations Theory, illustrated in section 2, this article investigates how the information and communication function of CMCs are perceived by local stakeholders in the communities where they operate. Section 3 presents the adopted research design, while Section 4 presents and discusses the research results. Implications for CMCs sustainability and for further advancement in the research area of PAVs are drawn in Section 5. 


\section{Social Representations: A Communication Perspective}

In this article, information and communication are considered in their different typologies of acquired, transmittable, and sharable flows, according to (i) the location of the sender of the message (inside or outside the community); and (ii) the direction of the message in relation to the local community (within or across its boundaries). This approach is inspired by the work of Heeks (2002), who suggested an informationcentred perspective, adopted an information chain approach to explain resources movements and directions in relation to telecentres, and studied resources brought in and taken out of the community (Heeks \& Kanashiro, 2009).

When communication happens, messages are exchanged, decoded and negotiated among individuals of a given social group. The negotiation of messages and meanings among social groups contributes to the groups' construction of a system of reference to understand reality, to make it meaningful, and to permit the repetition of the communication act. A communication perspective, then, lays at the basis of the theory of Social Representations (Moscovici, 1961), defined as:

"systems of values, ideas and practices with a twofold function; first, to establish an order which will enable individuals to orientate themselves in their material and social world and to master it; and secondly to enable communication to take place among the members of a community by providing them with a code for social exchange and a code for naming and classifying unambiguously the various aspects of their world and their individual and group history" (pp. IX-XIV).

This perspective was adopted to study the perception that different stakeholders have of CMCs as information and communication enablers, as well as to investigate wider meanings of CMCs in Mozambique. Similarities and differences in local representations and narratives will be also investigated according to social groups' demographic characteristics. 


\section{Research Design}

This article reports on local stakeholders' perceptions of the information and communication function of CMCs in Mozambique. In particular, it will analyse:

(1) Whether different social groups perceive CMCs as information and communication enablers;

(2) Which the perceived directions of the message in relation to the local community (within or across its boundaries) are;

(3) Which one of the two components of CMCs is perceived to convey more information and communication flows;

(4) Whether different social groups, according to different demographics, have different perceptions of information and communication flows connected to CMCs.

The following sections will describe the research design implemented in the study.

\subsection{Selection of the Sample}

A sample of 10 CMCs out of the 34 in the country (Vannini \& Rega, 2012) was selected, and various criteria were chosen to make the sample as demonstrative of the Mozambican situation as possible:

(1) First, a geographic criterion was applied: one CMC per province of the country was included, mirroring their real distribution as rural (9 CMCs in the selected sample) and urban ones (1 CMC).

(2) The second criterion applied was ownership type: CMCs in the country are managed mostly by local associations (7 included in the sample), as well as by the Government through the Institute of Social Communication of Mozambique 
(2 included in the sample), and by religious institutions (1 included in the sample, managed by an Order of Catholic Sisters).

(3) The year of foundation of the venue was also taken into account: some CMCs in Mozambique were built at the very beginning of the UNESCO programme, and some did exist even before as either stand-alone community radios or telecentres (in our sample, the oldest community radio station was established in 2000, and the oldest telecentre in 2001); some CMCs were established only as recently as 2010 by the MCT (1 included in the sample).

(4) The last criterion applied aimed to balance CMCs according to their variety of services, including both fully functional and more deficient ones. While all the centres had benefitted of the Internet in their past, and staff members still had ways to connect in most of the cases, only 2 CMCs offered access to the Internet to the public at the time of data collection (March-April 2011). This reflects the situation of Internet access of CMCs in the country. Out of 34 venues, all of them surveyed by the researchers by telephone during the month of January 2013, only 8 (23.5\% of the total) declared to offer the Internet connection to the public. In the survey, 4 of the centres included in this research declared to offer the Internet connection to the public (40\%). However, only 2 CMCs of the sample really offered the Internet connection to the public when researchers visited them (20\%).

In addition, the majority of the venues had offline digital libraries and produced newspapers ("newspaper on the wall”), and some offered services that were not initially foreseen by the original model (i.e.: language courses, cinema shows, etc.). There against, Community Radio programs and photocopies were common services offered by all CMCs, while basic computer courses were present in 7 
out of the $10 \mathrm{CMCs}$ included in the sample (for a detailed map of the services offered in each CMC included in the sample, see Rega et al., 2011).

\subsection{Data Collection}

During March and April 2011, 6 researchers undertook three different field trips to run semi-structured interviews: one to the Southern provinces of the country (Inhambane, Gaza, and Maputo), one to the Central provinces (Tete, Manica, Sofala and Zambezia), and one to the Northern ones (Cabo Delgado, Nampula and Niassa).

In determining the sample pool size, the initial goal was to interview a sample of the population that was as representative of the local interactions (or non-interactions) with the CMC. The intention was to interview, for each centre, 5 members of the local staff (both paid collaborators and volunteers), 10 users, and 10 members of the community who did not use the facility (non-users). During the fieldwork, however, it became clear that non-users were not illustrative of the local population: Community Radios are, in fact, a very pervasive medium within the communities, and pure nonusers were rare. Thus, the final subject pool utilized was of 230 interviews, divided as follows:

- 57 staff members: individuals from the community that were working or volunteering at the $\mathrm{CMC}$;

- 93 CMC whole users: people using both components of the CMC (telecentre and community radio);

- 72 Radio-only users: individuals who listened and interacted with the radio, but never used the telecentre component;

- 8 Non-users: people in the community who never listened to the radio nor accessed the telecentre. 
Different interview protocols, corresponding to different interviewee categories, have been prepared by the researchers, by following a semi-structure interview approach (Harcourt, 2006). The interview protocols were intended to investigate values, ideas and practices (Moscovici, 1961), ultimately social representations, that interviewees attribute to CMCs. The protocol was organised into four main parts, as shown in the table 1:

Table 1 - Interview protocols sections

\begin{tabular}{|l|l|}
\hline About the interviewee & $\begin{array}{l}\text { Demographics } \\
\text { Interviewee experience in the CMC } \\
\text { Interviewee exposure to media and ICTs }\end{array}$ \\
\hline CMC identity & $\begin{array}{l}\text { CMC history, structures, and goals } \\
\text { Benchmark, models, rites and future plans of the CMC } \\
\text { Services offered by the CMC }\end{array}$ \\
\hline $\begin{array}{l}\text { Community and the } \\
\text { CMC }\end{array}$ & $\begin{array}{l}\text { The CMC and the Community } \\
\text { People working at the venue } \\
\text { People visiting the venue }\end{array}$ \\
\hline Perception & Perception of the CMC and ICTs \\
\hline
\end{tabular}

Interviews lasted from a minimum of 7:08 minutes to a maximum of 2 hours and 14 minutes, depending on the category of interviewee: non-users interviews lasted less, due to the competence of the interviewees on the topic, and staff members' interviews lasted more, due to the complexity of their relation and interaction with the social object at stake. Overall, researchers collected 109 hours and 19 minutes of recorded interviews.

\subsection{Data Analysis}

Each interview audio file was named by indicating: location where the interview took place, interviewee category, number of interviewee for that category in the given CMC (e.g.: “Cuamba_Staff2” stays for CMC of Cuamba, second staff member interviewed). Then, interviews were transcribed and coded. A quali-quantitative content analysis approach was chosen (Krippendorff, 2003), with a first bottom-up phase 
leading to a preliminary explorative analysis done by using paper and pencil on a small number of interviews. This explorative analysis aimed to identify thematic areas and recurrent topics. At this point, a first formalization of the interpretative model was drawn. To manage the large body of data, a software for qualitative content analysis (NVivo, version 9.2) was used. A second phase of analysis included a top-down approach, moving from the first formalization of the interpretative model built. During the coding process, the interpretative model was continuously refined, in a constant shift between a top-down and a bottom-up approach meant to let the data "talk".

The result of this coding process is an interpretative model structured in eighteen different macro-themes, each of which opening up into sub-themes. While two of the macro-themes refer to people, the remaining sixteen inform the complex social representation system of CMCs in Mozambique. The structure of the model is presented in table 2:

Table 2 - CMCs interpretative model based on content analysis

\begin{tabular}{|c|c|c|c|}
\hline Values & Ideas & Practices & People \\
\hline $\begin{array}{l}\text { Benefits of CMC } \\
\text { Benefits of ICTs } \\
\text { Motivation to work } \\
\text { in CMC } \\
\text { Motivation to use } \\
\text { CMC } \\
\text { Reasons not to use } \\
\text { CMC }\end{array}$ & $\begin{array}{l}\text { Definitions of CMC, } \\
\text { Community Radio, } \\
\text { Telecentre, } \\
\text { computer, Internet } \\
\text { Improvements } \\
\text { needed in/challenges } \\
\text { for the CMC } \\
\text { Local denomination } \\
\text { of CMC } \\
\text { Services of the } \\
\text { Community Radio } \\
\text { Services of the } \\
\text { Telecentre } \\
\text { Services working } \\
\text { properly }\end{array}$ & $\begin{array}{l}\text { Community } \\
\text { involvement/support } \\
\text { in the CMC } \\
\text { Promotion of CMC } \\
\text { Synergies the CMC } \\
\text { is able to create } \\
\text { Usages of the } \\
\text { Community Radio } \\
\text { Usages of the } \\
\text { Telecentre }\end{array}$ & $\begin{array}{l}\text { Interviewee media } \\
\text { exposure } \\
\text { Staff Training }\end{array}$ \\
\hline
\end{tabular}


This article reports on the discourses related to interviewees’ perceptions on information and communication flows: in the model, their discourses are part of the macro-theme "Benefits of CMC", which refers to the values component of social representations of Mozambican CMCs. As mentioned in Section 2, information and communication flows were divided into three types, according to the location of the sender (inside or outside the community), and to the direction of the message (within or across the boundaries of the community). These three types of flows will be referred to as "inbound", “outbound”, and "shared within” information and communication flows:

(1) Inbound: includes all discourses about information and communication flows where the interviewee refers to information and communication received by the community from the outside. The information is, therefore, generated outside the community, and the CMC receives it and re-transmits it to the community. E.g.: international and national news, weather forecasts, etc.;

(2) Outbound: includes all statements referring to information and communication that is produced in and sent out from the community. E.g.: information the community sends to other villages and cities of the same region, or to governmental institutions;

(3) Shared within the community: includes all information, communication and knowledge that the community produces and shares within its boundaries. E.g.: lost and found announcements, advertisements of products, facts concerning community members, etc.

All discourses referring to generic benefits of CMCs in terms of information and communication that did not specify the direction and sender of the stream of information, and where the interviewee did not argue or explain their opinion, where excluded from this analysis. 
Differences of perceptions of CMCs information and communication flows by distinct social groups were investigated by performing a further analysis that considered socio-demographic variables:

- Interviewees’ category: staff members, users of both components of CMCs (UCMC), users of the radio component only (U-RC), people of the community who do not use the CMC (non-users);

- Interviewees’ education level: primary school, secondary school, high school, university, or no formal education received;

- Interviewees’ age: 10-19 years old, 20-29 years old, 30-39 years old, 40 or older.

According to the afore-mentioned variables, the general sample of interviewees is distributed as shown in Tables 3 and 4:

Table 3 - Interviewees according to their category and age groups

\begin{tabular}{|c|c|c|c|c|c|c|c|c|c|c|c|c|c|}
\hline & \multicolumn{12}{|c|}{ AgeGroup } \\
\hline & & \multicolumn{2}{|c|}{$10-19$} & \multicolumn{2}{|c|}{$20-29$} & \multicolumn{2}{|c|}{$30-39$} & \multicolumn{2}{|c|}{40 or +} & \multicolumn{2}{|l|}{ na } & \multicolumn{2}{|c|}{ Total } \\
\hline & & $\%$ & \# & $\%$ & \# & $\%$ & \# & $\%$ & \# & $\%$ & \# & $\%$ & $\#$ \\
\hline \multirow{4}{*}{ Category } & Staff & $9,8 \%$ & 6 & $25,5 \%$ & 25 & $32,4 \%$ & 12 & $43,8 \%$ & 14 & - & - & $24,8 \%$ & 57 \\
\hline & User CMC & $59,0 \%$ & 36 & $36,7 \%$ & 36 & $35,1 \%$ & 13 & 21,9\% & 7 & $50,0 \%$ & 1 & $40,4 \%$ & 93 \\
\hline & User RC & $26,2 \%$ & 16 & $35,7 \%$ & 35 & $27,0 \%$ & 10 & $31,3 \%$ & 10 & $50,0 \%$ & 1 & $31,3 \%$ & 72 \\
\hline & Non-User & 4,9\% & 3 & $2,0 \%$ & 2 & $5,4 \%$ & 2 & $3,1 \%$ & 1 & - & & $3,5 \%$ & 8 \\
\hline \multicolumn{2}{|c|}{ Totale } & $100,0 \%$ & 61 & $100,0 \%$ & 98 & $100,0 \%$ & 37 & $100,0 \%$ & 32 & $100,0 \%$ & 2 & $100,0 \%$ & 230 \\
\hline
\end{tabular}


Table 4 - Interviewees according to their Category and Education

\begin{tabular}{|c|c|c|c|c|c|c|c|c|c|c|c|c|c|c|c|}
\hline & \multicolumn{12}{|c|}{ Education } & & \\
\hline & & \multicolumn{2}{|c|}{$\begin{array}{c}\text { Did not go } \\
\text { to school }\end{array}$} & \multicolumn{2}{|c|}{ Primary } & \multicolumn{2}{|c|}{ Secondary } & \multicolumn{2}{|c|}{ High } & \multicolumn{2}{|c|}{$\begin{array}{c}\text { Universit } \\
\mathbf{y}\end{array}$} & \multicolumn{2}{|l|}{ na } & \multicolumn{2}{|c|}{ Totale } \\
\hline & & $\%$ & $\#$ & $\%$ & $\#$ & $\%$ & $\#$ & $\%$ & \# & $\%$ & \# & & \# & $\%$ & $\#$ \\
\hline \multirow{4}{*}{ Category } & Staff & & & $6,3 \%$ & 1 & $19,1 \%$ & 21 & $35,9 \%$ & 28 & $27,3 \%$ & 6 & $50,0 \%$ & 1 & $248 \%$ & 57 \\
\hline & \begin{tabular}{|l|} 
User \\
CMC \\
\end{tabular} & & & $18,8 \%$ & 3 & $48,2 \%$ & 53 & $38,5 \%$ & 30 & $31,8 \%$ & 7 & & & $40,4 \%$ & 93 \\
\hline & User RC & $100,0 \%$ & 2 & $75,0 \%$ & 12 & $29,1 \%$ & 32 & $24,4 \%$ & 19 & $27,3 \%$ & 6 & $50,0 \%$ & 1 & $31,3 \%$ & 72 \\
\hline & Non-User & & -7 & & - & $2,7 \%$ & 3 & $1,3 \%$ & 1 & $13,6 \%$ & 3 & & & $3,5 \%$ & 8 \\
\hline \multicolumn{2}{|c|}{ Totale } & $\mid 100,0 \%$ & 2 & $100,0 \%$ & 16 & $100,0 \%$ & 110 & $100,0 \%$ & 78 & $100,0 \%$ & 22 & $100,0 \%$ & 2 & $100,0 \%$ & 230 \\
\hline
\end{tabular}

\section{Results}

Beside information and communication, benefits of CMCs identified by the content analysis, include: learning, participation to the local socio-political life, edutainment activities, sparing money, being prepared for a better job, and having access to ICTs in general. Among them, information and communication flows constitute a key subject within the discourses related to the benefits of CMCs: in all cases, discourses were related to either the radio or the telecentre components of the venue, and never to the CMC as a whole.

Clearly, community radios are widely perceived as the place where communities' information and communication needs are mostly answered: community radios surface in $60.9 \%$ of the interviews that referred to information and communication, and telecentres only $7.4 \%$.

Information and communication discourses related to community radios are especially connected to inbound streams (46.1\%), and to flows that are shared inside community boundaries (34.3\%), thus picturing the radio as a mean for the members of the community to receive information and to communicate among themselves. The fact 
that only $4.8 \%$ of the discourses refers to outbound radio flows does not appear surprising, given the very nature of the medium: this kind of information stream would be expected to be related to the telecentre component of CMCs. Telecentres, however, do not seem to be perceived as information and communication enablers (only $7.4 \%$ of the interviewees mentioned it), and only as few as $0.4 \%$ of the discourses referred to their outbound communication function. Table 3 summarizes the aforementioned results.

Table 5 - Presence of discourses on information and communication flows in the interviews

\begin{tabular}{|r|r|r|}
\hline & $\%$ & $\#$ \\
\hline Community Radio & $60,9 \%$ & 140 \\
\hline Inbound & $46,1 \%$ & 106 \\
\hline Outbound & $4,8 \%$ & 11 \\
\hline Shared within & $34,3 \%$ & 79 \\
\hline Telecentre & $7,4 \%$ & 17 \\
\hline Inbound & $6,5 \%$ & 15 \\
\hline Outbound & $0,4 \%$ & 1 \\
\hline Shared within & $0,4 \%$ & 1 \\
\hline & $100,0 \%$ & 230 \\
\hline
\end{tabular}

The following sections will report on these results in detail, summarizing discourses about information and communication connected to either community radios or telecentres, and presenting socio-demographic differences among interviewees.

\subsection{Community radios: a democratic means to reinforce communities' social belonging}

Examples of radios perceptions as inbound information enablers include listening to international and national news: 
"The people here need to know what is happening. They need to get information about other part of the world", Chitima, Non-user3; 1

Getting information about government programmes:

"I think the radio created this space to let people stay close to the Government, and to let them know that not everything that is happening is against the population [...] and when the Government does something, they know that the Radio is going to broadcast it", Chiure, User3;

And getting developmental information about the relevant economic activities of the communities they serve:

"Most of all for the agriculture, it is one of the mass communication mean to tell when is the appropriate sowing time, when is the appropriate time for the harvest, what is going to happen in the land, what the peasant has to prepare for the land to be productive", Cuamba, Non-user 2.

In parallel, discourses of radios as means where social groups can share information and communicate with the other members of their community include numerous examples of announcements about relatives' illness and deaths, lost children, lost and found objects, and local commercials:

"It is different because, for example, before we had the radio, when someone died you had to send someone to notice it to the family, but now if someone dies, you can just transmit an announcement through the radio", Chitima, Staff1.

This function of the radio is indeed valuable for local people, for it is a fast, efficient, economic, and, in some cases, the only way to permit communication flows to be conveyed throughout the community:

"There are families that don't have other means to inform their relatives of an unhappy event [...] there are families that don't own a mobile phone [...] so they had to go to Xai-Xai", Chokwe, User6;

"It is important because without the radio there is no communication with the people [of the community] that live more distant", Cuamba, User8;

\footnotetext{
${ }^{1}$ The original statements have been translated from Portuguese into English by the authors of this paper.
} 
"We don't need phone balance anymore to call to someone [...] so with the radio we are transmitting the information to the community, it is important, they use a lot this service, it means that the radio is helping", Morrumbene, Staff5.

The few references to radios-related outbound communication flows are focused on the added value of being heard by nearby villages or by the main province city:

"... [before the radio was here] it was an extinguished world, you did not know whether we existed", Chiure, User8;

"There in Nampula they are listening that someone died in the island [...] so it is very important to have the radio", Ilha de Moçambique, User2.

Still, probably due to the nature of community radios as local entities, the possibility to use their communication potential as a means to actively participating to a bigger national or international reality is not contemplated.

Perceptions about radio shared within communication flows reveal how widely community radios are perceived as a means to act as social aggregators, and to respond to local information needs. Reasons for this success lays on the facts that they rely on oral communication and broadcast in local languages, thus permitting everybody to understand, and fitting the needs of a culture that is traditionally oral:

"Everyone pays attention because they have programs in Portuguese and programs in the Emakwa language, it is for that that the communities listen", Chiure, User 1.

\subsection{Telecentres: still not a window to the outside world}

Telecentres were created to serve as points of access to ICTs, and to address information and knowledge needs of underserved communities (Unwin, 2009). Thus, telecentres were expected to be mentioned as places to get informed from, and communicate with the outside world, via computers and the Internet.

As mentioned before, discourses about communication flows related to telecentres were surprisingly few, and referred more to telecentres’ inbound information value for the community than to its outbound information and communication potential: "In the computer there is a lot of information we can get", Cuamba, Non-user1. 
and

"Because people go there, in the Internet, and look for information, and inform the community, and the community is fulfilled in listening to the things that who went to [use] the Internet found", Cuamba, Non-User4.

Similarly, telecentres are not perceived as means to share information and to communicate within the community itself (possibility mentioned only by $0.4 \%$ of the interviewees). This datum reflects how access to the Internet is still a challenge not only for most of the individuals living in the communities, but also for the same CMCs: When the field work for this study was collected, only 2 out of 10 centres offered an Internet connection to the public, even if all of them used to have it in the past. CMCs are used as digital literacy training venues and centres to take photocopies, and not as much as places to freely access to computers and the web, activities that would permit an exchange of information and communication flows (see Rega et al., 2011; Vannini \& Rega, 2012).

\subsection{Socio-demographic analysis}

Differences in perceptions were analyzed also according to three sociodemographic variables: interviewees’ category, education level, and age group. While no substantial differences among the groups were found regarding interviewees’ age, the analysis on interviewees' category, education level, and CMC locations shed light on some interesting insights, which will be illustrated in the following sub-sections.

\subsubsection{Interviewees' category}

A deeper analysis on representations of CMCs connected to information and communication flows by different interviewees' typologies revealed an interesting insight on local staff members, who are far keener to recognize these flows as related to the community radio (78.9\%) than to the telecentre (5.3\%). Telecentres appear quite 
rarely in staff's discourses, and they are always connected to the inbound information potential of the telecentre, and never to shared-within-the-community or outbound communication flows: they are places where they can get information that they will, then, broadcast to the whole community through the radio.

Besides, staff members do not focus at all on the potential of computers and the Internet as means to communicate to an external world, which could open the horizons of rural communities: again, telecentres are considered only as centripetal information providers, and not as a way for the community to transmit their local knowledge outside its local and national limits.

As far as the information streams of the radio are concerned, staff members, $\underline{\mathrm{U}-}$ $\underline{\mathrm{CMC}}$, and $\underline{\mathrm{U}-\mathrm{RC}}$ attribute the same level of importance to the community radio: First, as an inbound information instrument; second, as an instrument to share information among the members of the community; and, finally, as a means with little potential for outbound communication. Non-users break this trend, by giving the same importance to inbound and shared communication flows, while they do not perceive the community radio as an outbound information means. Not surprisingly, non-users do not have any representation of telecentres as information and communication means. Table 4 presents the results divided by interviewees’ category.

Table 6 - Discourses on information and communication flows divided by interviewees’ category 


\begin{tabular}{|r|r|r|r|r|r|r|r|r|r|r|}
\hline & \multicolumn{2}{|c|}{ STAFF } & \multicolumn{2}{|c|}{ U-CMC } & \multicolumn{2}{c|}{ U-RC } & \multicolumn{2}{c|}{ NON-USERS } & \multicolumn{2}{c|}{ TOTAL } \\
\cline { 2 - 11 } & $\%$ & $\#$ & $\%$ & $\#$ & $\%$ & $\#$ & $\%$ & $\#$ & $\%$ & $\#$ \\
\hline Community Radio & $78,9 \%$ & 45 & $58,1 \%$ & 54 & $54,2 \%$ & 39 & $25,0 \%$ & 2 & $60,9 \%$ & 140 \\
\hline Inbound & $63,2 \%$ & 36 & $43,0 \%$ & 40 & $40,3 \%$ & 29 & $12,5 \%$ & 1 & $46,1 \%$ & 106 \\
\hline Outbound & $7,0 \%$ & 4 & $5,4 \%$ & 5 & $2,8 \%$ & 2 & $0,0 \%$ & 0 & $4,8 \%$ & 11 \\
\hline Shared within & $49,1 \%$ & 28 & $32,3 \%$ & 30 & $27,8 \%$ & 20 & $12,5 \%$ & 1 & $34,3 \%$ & 79 \\
\hline Telecentre & $5,3 \%$ & 3 & $10,8 \%$ & 10 & $5,6 \%$ & 4 & - & - & $7,4 \%$ & 17 \\
\hline Inbound & $5,3 \%$ & 3 & $8,6 \%$ & 8 & $5,6 \%$ & 4 & - & - & $6,5 \%$ & 15 \\
\hline Outbound & - & - & $1,1 \%$ & 1 & - & - & - & - & $0,4 \%$ & 1 \\
\hline Shared within & - & - & $1,1 \%$ & 1 & - & - & - & - & $0,4 \%$ & 1 \\
\hline & $100,0 \%$ & 57 & $100,0 \%$ & 93 & $100,0 \%$ & 72 & $100,0 \%$ & 8 & $100,0 \%$ & 230 \\
\hline
\end{tabular}

\subsubsection{Interviewees' education level}

When considering interviewees by education level, it is possible to see that people who completed only their primary education do not perceive telecentres as information and communication means at all, probably because they do not use computers and the Internet for this purpose. People with a university degree, instead, are the ones who mostly consider telecentres as a means to communicate and inform themselves and the others (18.2\% of the interviewees belonging to this education level). Moreover, they are the only ones who are able to recognize telecentres' value as outbound information enabler (4.5\% of them), while not attributing this value to the community radio at all: this is probably due to the fact that they have more choice, and more means to achieve this goal. Table 5 summarizes the results of the analysis, divided by respondents' education level.

Table 7 - Discourses on information and communication flows divided by interviewees’ education level 


\begin{tabular}{|r|r|r|r|r|r|r|r|r|r|r|r|r|r|r|}
\hline & \multicolumn{2}{|c|}{ Primary } & \multicolumn{2}{|c|}{ Secondary } & \multicolumn{2}{c|}{ High } & \multicolumn{2}{|c|}{ University $\mathbf{b}$ formal ed } & \multicolumn{2}{|c|}{ Total } \\
\cline { 2 - 13 } & $\%$ & $\#$ & $\%$ & $\#$ & $\%$ & $\#$ & $\%$ & $\#$ & $\%$ & $\#$ & $\%$ & $\#$ & $\%$ & $\#$ \\
\hline Community Radio & $43,8 \%$ & 7 & $60,9 \%$ & 67 & $65,4 \%$ & 51 & $59,1 \%$ & 13 & - & - & $100,0 \%$ & 2 & $60,9 \%$ & 140 \\
\hline Inbound & $31,3 \%$ & 5 & $45,5 \%$ & 50 & $52,6 \%$ & 41 & $40,9 \%$ & 9 & - & - & $50,0 \%$ & 1 & $46,1 \%$ & 106 \\
\hline Outbound & $6,3 \%$ & 1 & $2,7 \%$ & 3 & $9,0 \%$ & 7 & $0,0 \%$ & - & - & - & - & - & $4,8 \%$ & 11 \\
\hline Shared within & $12,5 \%$ & 2 & $34,5 \%$ & 38 & $42,3 \%$ & 33 & $22,7 \%$ & 5 & - & - & $50,0 \%$ & 1 & $34,3 \%$ & 79 \\
\hline Telecentre & - & - & $7,3 \%$ & 8 & $6,4 \%$ & 5 & $18,2 \%$ & 4 & - & - & - & - & $7,4 \%$ & 17 \\
\hline Inbound & - & - & $6,4 \%$ & 7 & $6,4 \%$ & 5 & $13,6 \%$ & 3 & - & - & - & - & $6,5 \%$ & 15 \\
\hline Outbound & - & - & - & - & - & - & $4,5 \%$ & 1 & - & - & - & - & $0,4 \%$ & 1 \\
\hline Shared within & - & - & $0,9 \%$ & 1 & - & - & - & - & - & - & - & - & $0,4 \%$ & 1 \\
\hline & $100,0 \%$ & 16 & $100,0 \%$ & 110 & $100,0 \%$ & 78 & $100,0 \%$ & 22 & $100,0 \%$ & 2 & $100,0 \%$ & 2 & $100,0 \%$ & 230 \\
\hline
\end{tabular}

\subsubsection{Interviewees' age group}

No substantial differences were found among interviewees' different age group: People older than 40 , the oldest group in the sample, focus on the community radio as information and communication means even more than the others, and do not consider the potential of the telecentre for this aim at all. Table 6 shows the discourses distributed by interviewees’ age group.

Table 8 - Discourses on information and communication flows divided by interviewees' age group

\begin{tabular}{|c|c|c|c|c|c|c|c|c|c|c|c|c|}
\hline & \multicolumn{2}{|l|}{ na } & \multicolumn{2}{|c|}{10 to 19} & \multicolumn{2}{|c|}{20 to 29} & \multicolumn{2}{|c|}{30 to 39} & \multicolumn{2}{|l|}{$40+$} & \multicolumn{2}{|c|}{ Total } \\
\hline & $\%$ & $\#$ & $\%$ & \# & $\%$ & \# & $\%$ & $\#$ & $\%$ & \# & $\%$ & $\#$ \\
\hline Community Radio & $50,0 \%$ & 1 & $52,5 \%$ & 32 & $63,3 \%$ & 62 & $54,1 \%$ & 20 & $78,1 \%$ & 25 & $60,9 \%$ & 140 \\
\hline Inbound & & & $42,6 \%$ & 26 & $46,9 \%$ & 46 & $35,1 \%$ & 13 & $65,6 \%$ & 21 & $46,1 \%$ & 106 \\
\hline Outbound & & & $1,6 \%$ & 1 & $5,1 \%$ & 5 & $5,4 \%$ & 2 & $9,4 \%$ & 3 & $4,8 \%$ & 11 \\
\hline Shared within & $50,0 \%$ & 1 & $21,3 \%$ & 13 & $34,7 \%$ & 34 & $40,5 \%$ & 15 & $50,0 \%$ & 16 & $34,3 \%$ & 79 \\
\hline Telecentre & & - & $11,5 \%$ & 7 & $5,1 \%$ & 5 & $13,5 \%$ & 5 & & & $7,4 \%$ & 17 \\
\hline Inbound & & & $11,5 \%$ & 7 & $4,1 \%$ & 4 & $10,8 \%$ & 4 & & & $6,5 \%$ & 15 \\
\hline Outbound & & & & - & $1,0 \%$ & 1 & & 7 & & & $0,4 \%$ & 1 \\
\hline Shared within & - & - & - & - & - & - & $2,7 \%$ & 1 & 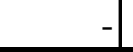 & & $0,4 \%$ & 1 \\
\hline & $100,0 \%$ & 2 & $100,0 \%$ & 61 & $100,0 \%$ & 98 & $100,0 \%$ & 37 & $100,0 \%$ & 32 & $100,0 \%$ & 230 \\
\hline
\end{tabular}

\subsection{Summary of the results}

The results of the analysis presented in this study show how information and communication flows are a key subject within the representations of CMCs in Mozambique. In particular, community radios appear as an essential means to answer 
information and communication needs of the local communities, while telecentres are still not widely perceived as such. Information and communication related to CMCs community radios are especially connected to inbound streams and to flows that are shared inside community boundaries. Telecentres, on their hand, do not seem to be perceived as enablers of an outbound communication.

The analysis of the different social groups interviewed shows how even staff members are far keener to recognize information and communication flows as related to community radios than to telecentres. Staff members do not seem to perceive the potential of computers and the Internet as means to communicate to an external world.

Local people with a university degree are the ones who mostly consider telecentres as a means to communicate and inform themselves and the others, and the only ones who are able to recognize telecentres’ value as outbound information enablers. Finally, no substantial differences were found among interviewees' different age group.

\section{Discussion and Conclusions}

This article presented a quali-quantitative content analysis of 230 interviews to local stakeholders of 10 CMCs around Mozambique. The study investigated local communities' perceptions about inbound, outbound and shared within communities information and communication flows connected to CMCs, and highlighted both that they are identified significantly more as information enablers through their radio component than through their telecentres, and that they are (still) not widely recognized as a means for the communities to actively participate as providers of information at a global level even by local staff members, who have generally more access to the Internet than the rest of the population. On the other hand, CMCs, through their radios, are considered as important communication means to share information within the 
communities they serve, where they act as social aggregators.

The little worth given to telecentres as information and communication enablers, especially towards the outside of the community, appears unexpected if matched up to the literature documenting the official model of CMCs (Creech, 2006; UNESCO, 2004). The lack of Internet access in CMCs might clearly have influenced their representations by local people. However, the sample selected for the study reflected the actual situation of Internet access of CMCs in the country. It would be worth investigating how the local communities have reinterpreted the role of CMCs according to a social representations perspective, as they might be perceived to have a different function, such as learning and training catalysts.

Throughout this fieldwork, no particular integration of mobile services in CMCs practices in Mozambique was observed. Yet, mobile phones are a fast-growing technology in Africa. Future research outlook should consider whether and how mobile phones support and change information and communication flows of the communities through CMCs, which uses and affordances they provide, and how local social representations are influenced by this new technology.

It is crucial that funding agencies take into consideration local perspectives and appropriation of CMCs while planning the future of these initiatives. Acknowledging local representations will guarantee CMCs sustainability, by better embedding them into the social context, and promoting an increased community ownership.

\section{Acknowledgments}

This study is part of the broader project RE-ACT (social REpresentations of community multimedia centres in Mozambique and ACTions for improvement), a joint research and cooperation project between the NewMinE Lab - New Media in Education Laboratory of the Università della Svizzera italiana (Lugano, Switzerland), and the 
Department of Mathematics and Informatics and the Centre of African Studies of the Universidade Eduardo Mondlane (Maputo, Mozambique). RE-ACT Project is funded by the Swiss National Science Foundation (SFNS) and the Swiss Agency for Development and Cooperation (SDC).

\section{References}

Creech, H. (2006). Evaluation of UNESCO’s Community Multimedia Centres. UNESCO. Retrieved from http://portal.unesco.org/pv_obj_cache/pv_obj_id_BE7A0D92CAAF9399898BB 8BE86A10234676C0500/filename/CMC+Evaluation_Final.pdf

Harcourt, B. E. (2006). Language of the Gun: Youth, Crime, and Public Policy. University of Chicago Press.

Heeks, R. (2002). i-development not e-development: special issue on ICTs and development. Journal of International Development, 14(1), 1-11. doi:10.1002/jid.861

Heeks, R., \& Kanashiro, L. L. (2009). Telecentres in mountain regions — A Peruvian case study of the impact of information and communication technologies on remoteness and exclusion. Journal of Mountain Science, 6(4), 320-330. doi:10.1007/s11629-009-1070-y

Krippendorff, K. H. (2003). Content Analysis: An Introduction to Its Methodology (2nd ed.). Sage Publications, Inc.

MCT. (2008, June 13). Programa Nacional de Centros Multimedia Comunitários, Ver 4.5. MCT, Republica de Moçambique.

Moscovici, S. (1961). La Psychanalyse, son image et son public. Presses Universitaires de France - PUF. 
Rega, I., Cantoni, L., Vannini, S., David, S., Baia, A., \& Macueve, G. (2011). Community Multimedia Centres in Mozambique: a Map. (White Paper No. 1.0). Lugano: NewMinE Lab. - USI. Retrieved from http://www.newmine.org/publications-2/working-papers/white-papercommunity-multimedia-centres-in-mozambique-a-map

UNESCO. (2004). Scale up Initiative for Community Multimedia Centres in Mozambique. UNESCO.

Unwin, T. (Ed.). (2009). ICT4D: Information and Communication Technology for Development (1st ed.). Cambridge University Press.

Vannini, S., \& Rega, I. (2012). Inbound and Outbound Information and Communication flows: Perspectives from Community Multimedia Centres in Mozambique. In CIRN 2012 Community Informatics Conference: “Ideals meet Reality” Monash Centre. Presented at the CIRN 2012, Prato, Italy.

WSIS. (2003, December 12). Declaration of Principles: Building the Information Society: a global challenge in the new Millennium. WSIS-03/GENEVA/DOC/4E. Retrieved from http://www.itu.int/wsis/docs/geneva/official/dop.html 